\title{
Operación de Bascom para el tratamiento de la enfermedad pilonidal sacrococcígea abscedada. Experiencia inicial
}

\author{
Alejandro Barrera E. ${ }^{1}$, Sebastián Pradenas B. ${ }^{1}$, Guillermo Bannura C. ${ }^{1}$, \\ Felipe Illanes F. ${ }^{1}$, Cristian Gallardo V. ${ }^{1}$, Baldo Rinaldi C. ${ }^{1}$, \\ Andrea Madariaga R. ${ }^{1}$ y Constanza Espinoza R. ${ }^{1}$
}

'Servicio y Departamento de Cirugía, Hospital Clínico San Borja Arriarán, Facultad de Medicina, Universidad de Chile, Santiago, Chile.

Recibido el 5 de junio de 2017 y aceptado para publicación el 26 de julio de 2017

Correspondencia a: Dr. Alejandro Barrera E. alejandro.barrera@gtdmail.com

\section{Bascom operation for the treatment of abscessed pilonidal sacrococcygeal disease. Initial experience}

Introduction: One of the form of presentation of the sacral coccygeal pilonidal disease is the abscess, for this cases there are various treatment alternatives. Objective: We present our experience with Bascom technique for the treatment of pilonidal abscess. Material and Method: Prospective, consecutive, nonrandomized series. It includes all patients older than 15 years who have an abscess or mass discharge at the time of surgery. Results: The series consists of 10 patients, 7 males. They are not risk factors recognized in the literature as risk of pilonidal disease. Eight patients completely better within a maximum period of 18 days and two with persistent discharge by what is considered treatment failure. They were subjected to a second surgery treatment with another technique with good results. Conclusion: Bascom's technique is simple, safe and offers a $80 \%$ cure in a short period of healing.

Key words: sacrococcygeal pilonidal disease; Bascom; pilonidal abscess.

\section{Resumen}

Introducción: Una de las formas de presentación de la enfermedad pilonidal sacrococcígea (EPSC) es el absceso, para el que existen distintas alternativas de tratamiento. Objetivo: Presentar nuestra experiencia con la técnica de Bascom para el tratamiento de la EPSC abscedada. Material y Método: Serie prospectiva, consecutiva y no aleatoria. Incluye todos los pacientes mayores de 15 años que presentan un absceso o supuración masiva al momento de la cirugía. Resultados: La serie corresponde a 10 pacientes, 7 de género masculino. No se encuentran los factores de riesgo reconocidos por la literatura como riesgo de EPSC. Ocho pacientes mejoran completamente en un período máximo de 18 días y 2 presentan supuración persistente, por lo que se considera fracaso del tratamiento. Estos son sometidos a una segunda cirugía con otra técnica, con curación de la enfermedad. Conclusión: La técnica de Bascom es sencilla, segura y ofrece una curación de un $80 \%$ en un período corto de cicatrización.

Palabras clave: enfermedad pilonidal sacrococcígea; Bascom; absceso pilonidal.

\section{Introducción}

La enfermedad pilonidal sacrococcígea (EPSC) es una condición adquirida cuya fisiopatología aún no es completamente comprendida. Es una enfermedad bastante frecuente, que afecta principalmente a hombres jóvenes laboralmente activos ${ }^{1-3}$, aunque reportes nacionales anteriores señalaban una incidencia mayor en mujeres.
Los resultados de la cirugía de la EPSC se miden por el tiempo de cicatrización, la tasa de complicaciones y la recurrencia. Se han descrito muchas técnicas para el tratamiento de esta patología, desde resecciones simples, con cierre primario o no de la zona cruenta, hasta el avance de colgajos ${ }^{4-7}$. No existe hasta hoy un elevado nivel de evidencia para definir el mejor tratamiento de la EPSC, aunque una revisión reciente sugiere que las técnicas cerradas 
con una herida fuera de la línea media deben ser el estándar de tratamiento electivo en la $\mathrm{EPSC}^{7}$.

Entre las formas de presentación de urgencia, se destaca el absceso, el cual generalmente se trata con un drenaje, dejando el manejo definitivo en forma diferida, o con técnicas abiertas, que dejan una herida que cicatriza por segunda intención en plazos variables $^{8-10}$, agregando la necesidad de curaciones frecuentes y la posibilidad de que esto no tenga un resultado estético satisfactorio.

El objetivo de este trabajo es mostrar nuestra experiencia en el manejo del absceso pilonidal con la técnica de Bascom ${ }^{8}$.

\section{Material y Método}

Desde el año 2001 se lleva un registro prospectivo de los pacientes operados en forma consecutiva por EPSC en el Servicio de Cirugía del Hospital Clínico San Borja Arriarán. Durante el período comprendido entre enero del año 2001 y diciembre del año 2007 los pacientes con absceso o supuración evidente, calificada como masiva por el cirujano al momento de la intervención, eran drenados o tratados en diferido. Desde el año 2007 hasta la actualidad, los pacientes que al momento de la cirugía electiva presentaban estas condiciones fueron operados con la técnica de Bascom.

Los criterios de inclusión fueron pacientes mayores de 15 años con un absceso sacrococcígeo y/o secreción purulenta calificada como abundante por el cirujano en el pabellón previo a la intervención. Se excluyeron los pacientes que eran intervenidos por una recidiva.

Todos los pacientes fueron operados en posición de navaja sevillana con retracción de los glúteos con esparadrapos adhesivos. Se utilizó anestesia regional de tipo espinal. En primer lugar se realizó la resección de los poros dilatados (pits) hasta alcanzar la cavidad del absceso (Figura 1). Posteriormente, se realizó resección del trayecto fistuloso en caso de que existiese (Figura 2), seguido del curetaje de la zona y, finalmente, de un drenaje lateral alejado de la línea media (Figura 3). Se suturaron las heridas de la resección de los poros dilatados y del trayecto fistuloso dejando abierta la zona de drenaje (Figura 4). $\mathrm{Al}$ alta se mantuvieron curaciones diarias de la zona del drenaje para el cierre por segunda intención.

Se analizaron los datos demográficos, el tiempo operatorio, el tiempo de cicatrización, la persistencia o recurrencia y el seguimiento a corto y largo plazo.

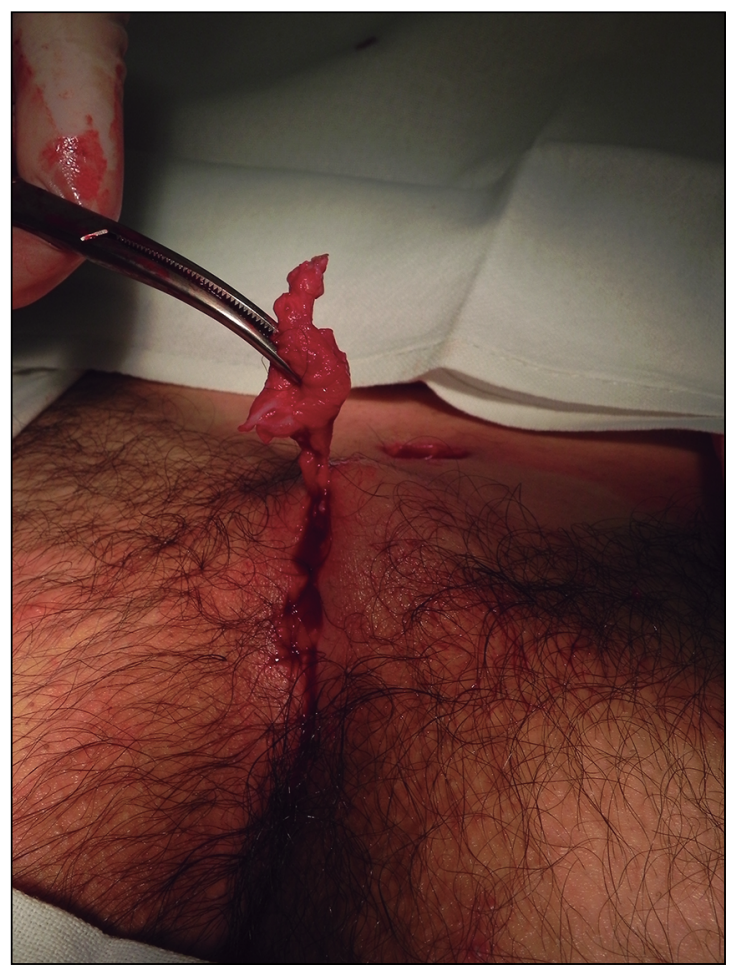

Figura 2. Resección de poros dilatados y trayecto fistuloso.
Figura 1. Resección de poros dilatados (pits).

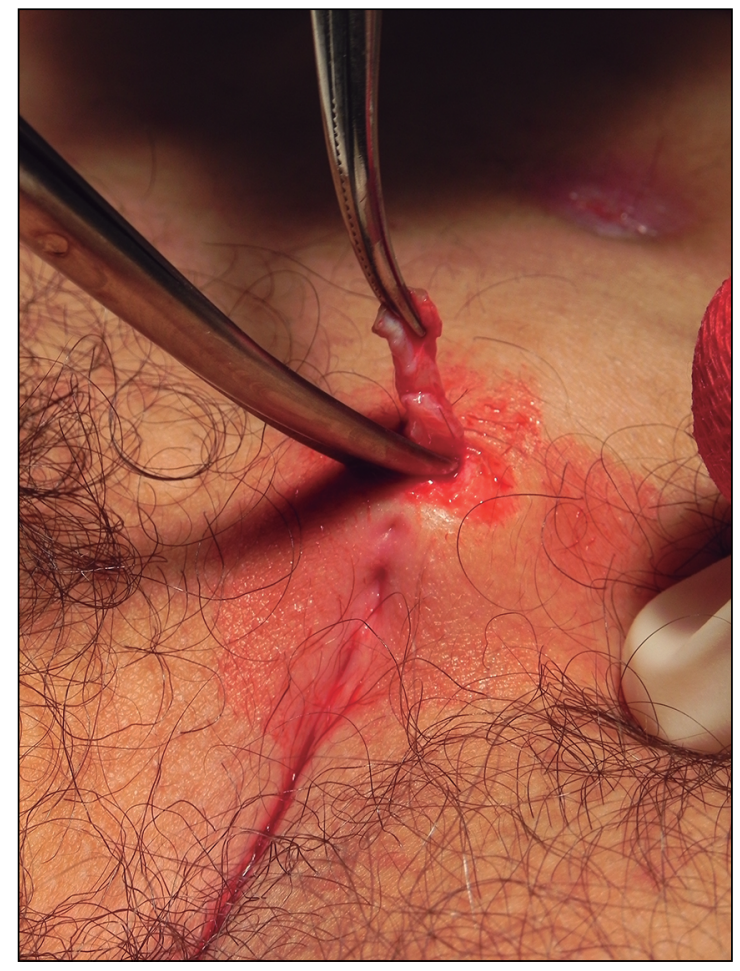




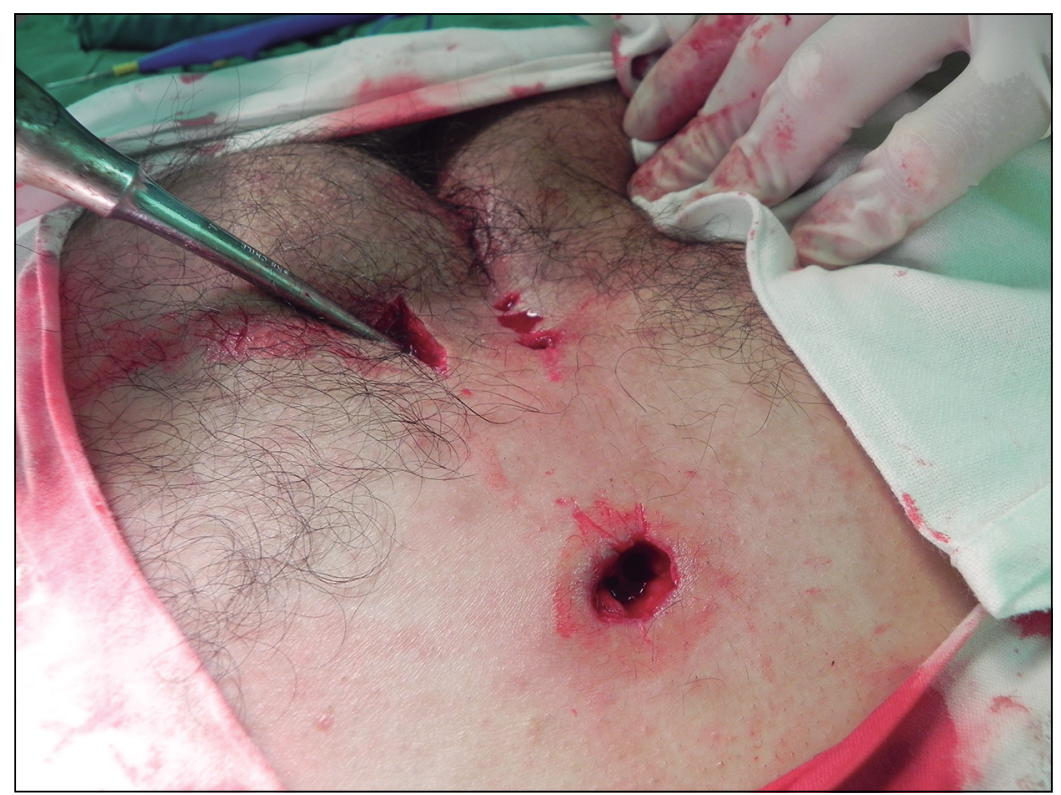

Figura 3. Drenaje lateral.

Figura 4. Resultado final.

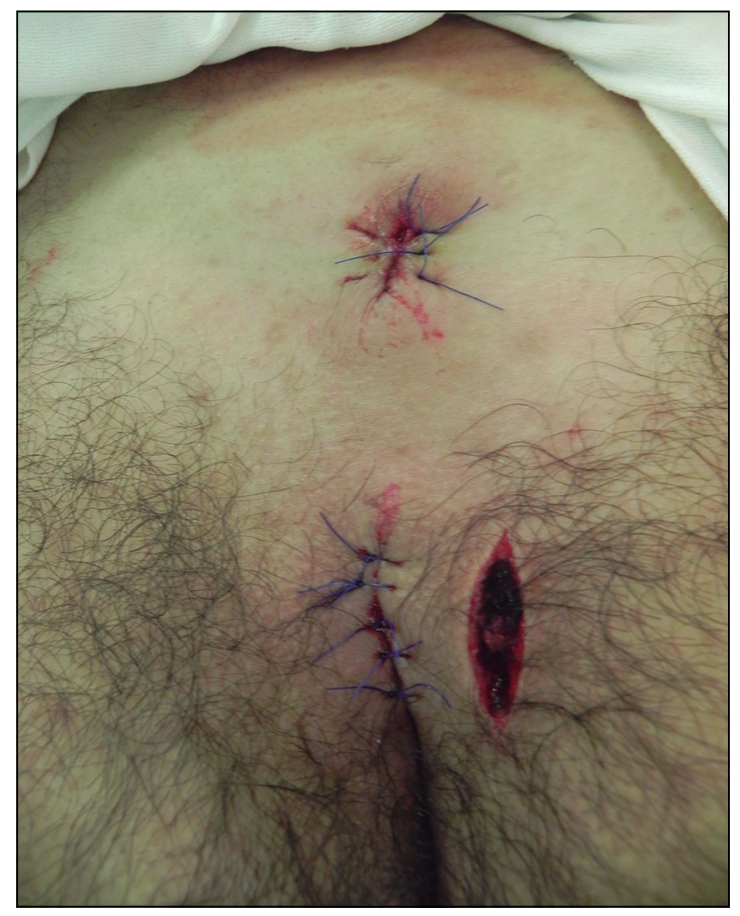

\section{Resultados}

La serie corresponde a 10 pacientes, 7 de género masculino y 3 de género femenino. Todos presentaban un absceso o supuración masiva evidente al momento de la cirugía. El índice de masa corporal promedio de los pacientes de esta serie fue de 28,1 (extremos de 24 y 31). Solo uno de los pacientes fue catalogado como hirsuto por el cirujano tratante.

El período sintomático promedio fue de 12 meses (extremos 3-24) y todos los pacientes presentaban al menos un episodio previo de absceso.

La cirugía se realizó de forma ambulatoria en 9 pacientes. Uno tuvo que permanecer hospitalizado por $24 \mathrm{~h}$ para el manejo del dolor y fue dado de alta al día siguiente sin inconvenientes.

Ocho pacientes curaron su enfermedad y no han presentado recidiva hasta el momento, con seguimientos entre 6 meses y 5 años. El tiempo promedio de curación de las heridas, incluyendo la lateral, fue de 14 días con extremos de 7 y 18. Los 2 pacientes restantes presentaron supuración mantenida por el drenaje lateral, por lo que se consideró su enfermedad como persistente. Ambos fueron reintervenidos a los 6 meses de la cirugía original, uno con un colgajo de Limberg y el segundo con una operación de Karydakis con buenos resultados inmediatos y alejados.

\section{Discusión}

La EPSC es una entidad frecuente, aunque no se conoce su real incidencia. Una de las dificultades para establecerla es que en muchas series se incluyen, además de los pacientes que presentan manifestaciones inflamatorias evidentes como eritema, supuración aguda o crónica y dolor asociado a aumento de volumen, aquellos que presentan un seno en la región sacrococcígea o poros dilatados, sin manifestaciones inflamatorias no masa evidente ${ }^{9}$. En estos casos, una ecografía de partes blandas, con un transductor de alta resolución puede determinar la presencia de una lesión seudoquística en el celular subcutáneo, con lo que se certifica la enfermedad. No existe evidencia de que la presencia del seno pilonidal sin inflamación como factor anatómico aislado sea predisponente de la aparición de inflamación a mediano plazo $^{10}$.

Entre los factores de riesgo para la aparición de la enfermedad sintomática se reconocen el género masculino, la obesidad y el hirsutismo, aunque ni en esta serie, ni en nuestra serie histórica esto ha sido demostrado ${ }^{11}$.

Para su tratamiento se han propuesto muchas técnicas, desde simples resecciones dejando lechos cruentos hasta el avance de colgajos ${ }^{12,13}$. No existe consenso ni evidencia para definir el mejor tratamiento. La presencia de secreción purulenta o un 
absceso al momento de la consulta en la mayoría de los casos es tratada con un drenaje, dejando la resolución definitiva en diferido. En estos casos la técnica de Bascom nos ofrece una alternativa sencilla, con buenos resultados a corto plazo. Otras alternativas, como la marsupialización o la técnica de McFee, pueden lograr el mismo objetivo, sin embargo, los plazos de curación de la herida son superiores ${ }^{14}$ a los mostrados en esta comunicación, lo que muestra las ventajas de esta técnica.

El seguimiento en la mayoría de los pacientes fue superior al año sin recidivas. Además, en los pacientes que fueron reintervenidos, la supuración fue mantenida, por lo que fueron considerados con una enfermedad persistente, lo que agregaría la potencial ventaja de identificar rápidamente aquellos pacientes que curaron su enfermedad. Al ser esta una técnica sencilla en los casos de persistencia y/o recurrencia uno puede plantear las técnicas habituales de manejo de la enfermedad, como es la operación de Karydakis, reservando los colgajos para los casos más complejos.

La principal desventaja de este trabajo es que se trata de una serie reducida de pacientes, pero pareciese, a la luz de los resultados, que es una técnica a tomar en cuenta en pacientes que se presentan con un absceso y/o secreción ya que no solo es una técnica sencilla, que se puede realizar de forma ambulatoria, sino que sus reducidos tiempos de cicatrización, de reinserción laboral y la baja tasa de recurrencia permiten realizarla con seguridad.

\section{Conclusión}

La operación de Bascom es una alternativa sencilla para el tratamiento del absceso pilonidal. El tiempo de convalecencia y sus tasas de curación hacen que sea preferible al drenaje simple y a la marsupialización en el manejo de la EPSC asociada a un absceso y/o secreción activa.

\section{Responsabilidades éticas}

Protección de personas y animales. Los autores declaran que los procedimientos seguidos se conformaron a las normas éticas del comité de experimentación humana responsable y de acuerdo con la Asociación Médica Mundial y la Declaración de Helsinki.

Confidencialidad de los datos. Los autores declaran que han seguido los protocolos de $\mathrm{su}$ centro de trabajo sobre la publicación de datos de pacientes.

Derecho a la privacidad y consentimiento informado. Los autores han obtenido el consentimiento informado de los pacientes y/o sujetos referidos en el artículo. Este documento obra en poder del autor de correspondencia.

\section{Conflicto de intereses}

Los autores declaran que no tienen conflictos de interés.

\section{Bibliografía}

1. Bannura G, Barrera A, Contreras J, Melo C, Soto D. Operación de Karydakis ambulatoria en el manejo de la enfermedad pilonidal sacrococcígea. Rev Chil Cir. 2009;61:256-60.

2. Eftaiha MS, Abcarian H, Weinstein M, Rosemberg I, Salvati EP. The dilemma of pilonidal disease. Dis Colon Rectum 1977;20:278-86.

3. Da Silva JH. Pilonidal cyst. Cause and treatment. Dis Colon Rectum 2000;43:1146-56.

4. Petersen S, Koch R, Stelzner S, Wendlandt T, Ludwig K. Primary closure techniques in chronic pilonidal sinus. Dis Colon Rectum 2002;45: 1458-67.

5. Enríquez-Navascues JM, Emparanza JI Alkorta M, Placer C. Meta-analysis of randomized controlled trials comparing diffe- rent techniques with primary closure for chronic pilonidal sinus. Tech Coloproctol. 2014;57:863-72.

6. Lee P, Raniga S, Biyani D, Watson A, Faragher I, Frizelle F. Sacrococcygeal pilonidal disease. Colorectal Dis. 2008;10:639-52.

7. McCallum I, King P, Bruce J. Healing by primary closure versus open healing after surgery for pilonidal sinus: Systematic review and meta-analysis. BMJ

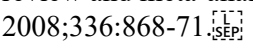

8. Bascom J. Pilonidal disease: Origin from follicles of hair and results of follicle removal as treatment. Surgery 1980;87:567-72

9. Kanat B, Sözen S. Disease that should remember. Sacrococcigeal pilonidal sinus disease and short history. World J Clin Cases 2015;3:876-9.

10. Doll D, Friederichs J, Boulesteix A, Düsel W, Fend F, Petersen S. Surgery for asymptomatic pilonidal sinus disease. Int J Colorectal Dis. 2008;23:839-44.
11. Bannura G, Cumsille M, Contreras J, Barrera A, Soto D, Melo C, et al. Obesidad e hirsutismo como factores predisponentes de la enfermedad pilonidal sacrococcígea. Rev Chil Cir. 2007;59:13641.

12. Guner A, Boz A, Ozkan O, Ileli O, Kece C, Reis E. Limberg flap versus Bascom cleft lift techniques for sacrococcygeal pilonidal sinus: Prospective, randomized trial. World J Surg. 2013;37:2074-80.

13. Nordon I, Senapati A, Cripps N. A prospective randomized controlled trial of simple Bascom's technique versus Bascom's cleft closure for the treatment of chronic pilonidal disease. Am J Surg. 2009;197:189-92.

14. Inostroza G, García E, Yussem MJ, Mariangel P, Yussem P, Salazar A. Quiste pilonidal abscedado: tratamiento definitivo en un solo tiempo durante el episodio agudo, resultados a corto plazo. Rev Chil Cir. 2011;63:53-8. 